\title{
Direct observation of plasmonic index ellipsoids on a deep-subwavelength metallic grating
}

\author{
Liang Feng, ${ }^{1,2, *}{ }^{2}$ haowei Liu, ${ }^{1}$ and Yeshaiahu Fainman ${ }^{1}$ \\ ${ }^{1}$ Department of Electrical and Computer Engineering, University of California, San Diego, La Jolla, California 92093, USA \\ ${ }^{2}$ Department of Electrical Engineering, California Institute of Technology, Pasadena, California 91125, USA \\ *Corresponding author: Ifeng@ caltech.edu
}

Received 29 June 2011; accepted 21 July 2011;

posted 29 July 2011 (Doc. ID 149771); published 9 September 2011

\begin{abstract}
We constructed a metallic grating on a deep-subwavelength scale and tested its plasmonic features in visible frequencies. The deep-subwavelength metallic grating effectively acts as an anisotropic homogeneous uniaxial form-birefringent metal, exhibiting different optical responses for polarizations along different optical axes. Therefore, this form-birefringent metal supports anisotropic surface plasmon polaritons that are characterized by directly imaging the generated plasmonic index ellipsoids in reciprocal space. The observed plasmonic index ellipsoids also show a rainbow effect, where different colors are dispersively distributed in reciprocal space. (C) 2011 Optical Society of America
\end{abstract}

OCIS codes: $\quad 240.6680,310.6628,050.2555$.

\section{Introduction}

Optical anisotropy exists in many natural crystals such as calcite and quartz. It has been widely used in various applications including polarizers, phase compensators, modulators, and displays. Optical anisotropy refers to optical materials which possess polarizability that depends on the directions of propagation and polarization of electromagnetic fields. This phenomenon originates from atomic scale dipole moments that vary in space depending on the crystal lattice structure. The susceptibility and the corresponding polarizability of such media will vary in space depending on the propagation direction and the state of polarization of the optical fields. Consequently, such crystals possess indices of refraction that depend on the crystallographic lattice structure and in most general cases are described in reciprocal space by an index ellipsoid in three dimensions [1,2] Artificial structures called "form birefringence" are composed of space-variant polarizability of isotropic dielectrics with deep-subwavelength geometry [3] and have been also developed and used for various

0003-6935/11/3100G1-06\$15.00/0

(C) 2011 Optical Society of America applications to manipulate the polarization state of the electromagnetic fields in forms that are not possible with natural birefringent crystalline dielectrics [4-8]. For example, a deep-subwavelength GaAs grating enables large phase retardation for different polarized incident light [8].

Similarly, a wire grid polarizer made of a deep subwavelength metallic grating can be viewed as a form-birefringent metal, exhibiting different optical properties along different optical axes in a broad band of wavelengths: it acts as dielectrics when the polarization is along the grating vector, while it shows metal response when the polarization is perpendicular to the grating vector [9]. Form-birefringent metals have been recently used to realize negative refraction of the propagating optical fields because of unique hyperbolic dispersion properties associated with simultaneous combination of both metallic and dielectric polarizabilities $[10,11]$. Because of this complexity, interesting plasmonic properties, such as anisotropic propagation of surface plasmon polariton (SPP) waves, can be envisioned on deep-subwavelength metallic gratings. In this paper we characterize the plasmonic properties on a deep-subwavelength $\mathrm{Au}$ grating by directly imaging the corresponding index ellipsoids of SPPs. Section $\underline{2}$ describes the design of 
the deep-subwavelength Au grating as well as discusses the effective dielectric tensor of the grating. In Section 3 the simulated and measured plasmonic index ellipsoids are studied with unpolarized and polarized incidences, and the corresponding dispersion relation of SPPs is constructed. Conclusions are given in Section $\underline{4}$.

\section{Form-Birefringent Metal}

The form-birefringent metal is a metallic grating consisting of an array of Au stripes periodically arranged in the $x$ direction in a deep-subwavelength scale as schematically depicted in Fig. 1(a). The periodicity of the grating is $d=140 \mathrm{~nm}$, and the width and the thickness of the Au stripes are $a=100 \mathrm{~nm}$ and $h=55 \mathrm{~nm}$, respectively, much shorter than the wavelengths we study in this work. Because of the deep-subwavelength nature of the structure, all the diffraction orders fall into the evanescent regime. Therefore the deep-subwavelength metallic grating shows a uniform optical response without involving any diffraction. Its corresponding effective dielectric constant tensor can be described as

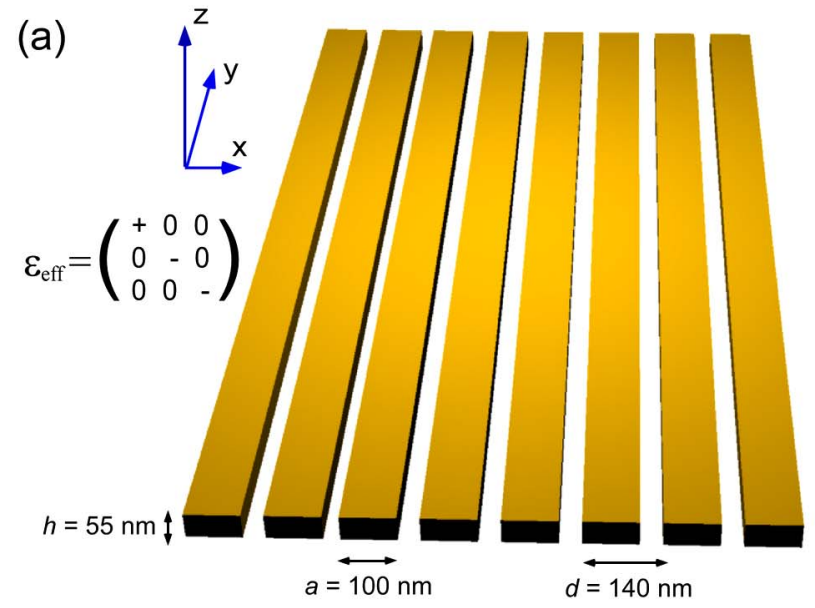

(b)

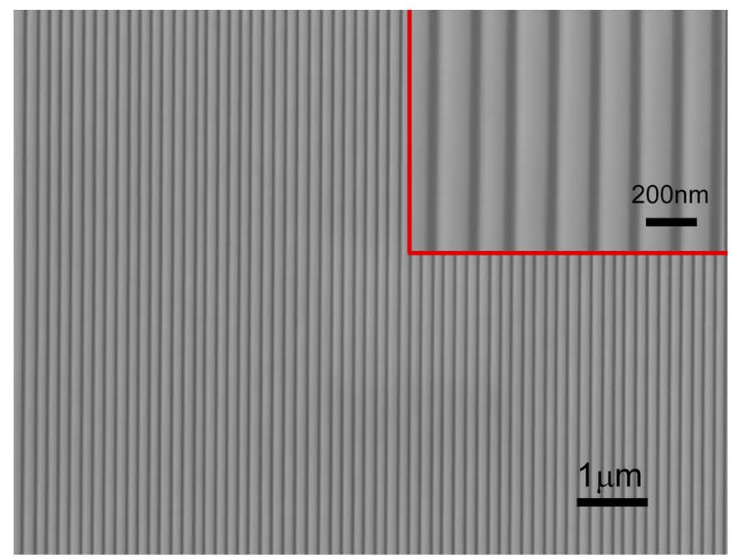

Fig. 1. (Color online) (a) Schematic of the designed deepsubwavelength $\mathrm{Au}$ grating with the parameters: $d=140 \mathrm{~nm}$, $a=100 \mathrm{~nm}$, and $h=55 \mathrm{~nm}$. (b) SEM micrograph of the fabricated nanostructure using FIB milling.

$$
\varepsilon_{\text {eff }}=\left(\begin{array}{ccc}
\varepsilon_{\|} & 0 & 0 \\
0 & \varepsilon_{\perp} & 0 \\
0 & 0 & \varepsilon_{\perp}
\end{array}\right),
$$

where $\varepsilon_{\|}$and $\varepsilon_{\perp}$ refer to polarization of optical fields parallel and perpendicular to the grating vector, respectively. Using the effective medium theory, the three diagonal elements are approximately expressed by [12]

$$
\varepsilon_{\|}=\frac{\varepsilon_{m} \varepsilon_{0}}{(1-f) \varepsilon_{m}+f \varepsilon_{0}}, \quad \varepsilon_{\perp}=f \varepsilon_{m}+(1-f) \varepsilon_{0},
$$

where $\varepsilon_{m}$ and $\varepsilon_{0}$ are the dielectric constants of metal (e.g., $\mathrm{Au}[13])$ and dielectric medium in the slits (e.g., air), respectively, and $f=a / d$ is the duty cycle of $\mathrm{Au}$ stripes. From Eq. (2), form birefringence can be clearly seen and the constructed grating can be considered as an anisotropic material: the grating behaves like dielectrics if polarization is along the $x$ direction $\left(\varepsilon_{x}=\varepsilon_{\|}>0\right)$, while it acts as a metal for polarization in $y$ and $z$ directions $\left(\varepsilon_{y}=\varepsilon_{z}=\varepsilon_{\perp}<0\right)$.

\section{Results and Discussion}

For experimental validations, we used focusedion-beam (FIB) milling to fabricate fine deepsubwavelength features shown in Fig. 1(a) using an Au film on a glass substrate. A $120 \mu \mathrm{m}$ thick glass slide was first cleaned using acetone in an ultrasonic bath, followed by deposition of $2 \mathrm{~nm} \mathrm{Ti}$ and $55 \mathrm{~nm} \mathrm{Au}$ using electron-beam evaporation. FIB milling was next applied to fabricate an array of air slits with the width of $40 \mathrm{~nm}$ and the periodicity of $140 \mathrm{~nm}$ along the $x$ direction through the Au film. The scanning electron microscope (SEM) micrograph of the fabricated structure is shown in Fig. 1(b).

The material birefringence of the fabricated $\mathrm{Au}$ grating is expected to be transferred to its supported SPPs. To validate the expected plasmonic anisotropy, the corresponding SPP modes on the deepsubwavelength $\mathrm{Au}$ grating are characterized using the reciprocal space representations $[14,15]$. The index ellipsoids of the excited SPP modes in the fabricated Au grating are characterized by analyzing reflection spectra at different elevation and azimuth angles simultaneously obtained from the glass substrate side of the samples.

Figure 2 shows the numerically simulated index ellipsoids of SPPs using the rigorous coupled wave analysis and experimentally measured ones on the deep-subwavelength $\mathrm{Au}$ grating at the wavelength around $540 \mathrm{~nm}$. In simulations [Fig. 2(a)], reflection intensities are plotted in a polar coordinate where the polar angle is the incident azimuth angle and the radial direction is the in-plane wave vector $k_{\|}$, corresponding to different elevation angles of incidence. In experiments, the desired wavelength was obtained from a broadband light source using a series of $10 \mathrm{~nm}$ bandpass interference filters in the spectral range of interest, i.e., from 540 to $680 \mathrm{~nm}$. An oil immersion microscope objective (MO) with a high 
(a)

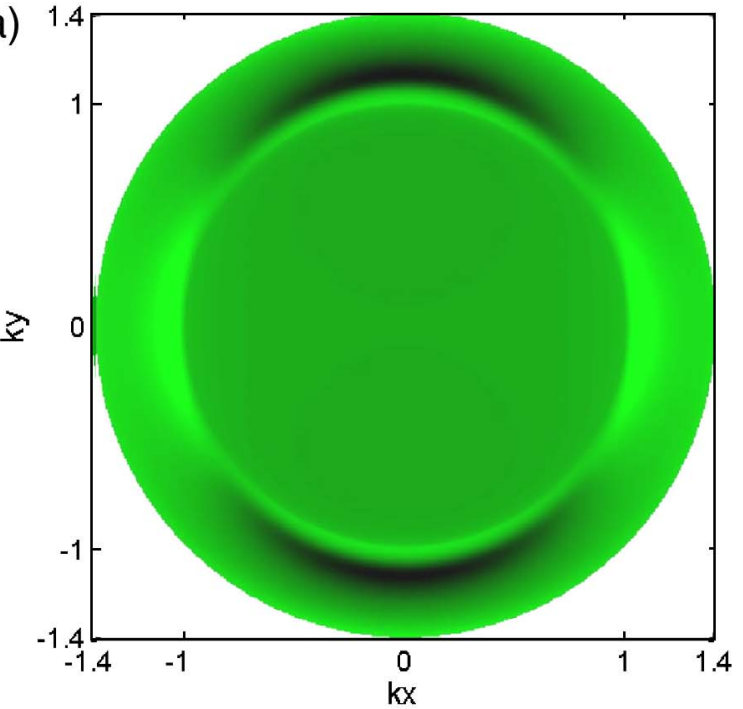

(b)

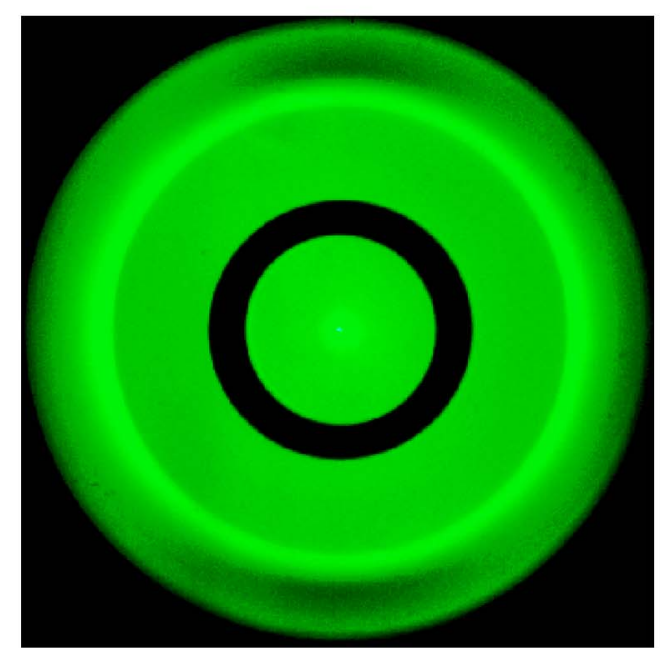

Fig. 2. (Color online) Plasmonic index ellipsoids at the wavelength of $540 \mathrm{~nm}$. (a) Simulated SPP's index ellipsoid in a representation of reflected power distribution in reciprocal space. (b) Experimental imaging of reflection from the deepsubwavelength Au grating in reciprocal space. The dark elliptical curves indicate the excitation of anisotropic SPP waves and are the corresponding SPP index ellipsoids.

numerical aperture $(\mathrm{NA}=1.4)$ was used to converge an unpolarized spherical wave onto the grating and code the wideband angular spectrum of the illumination function within the illumination circle. The Fourier transform of the reflection image was optically captured using an additional lens $[14,15]$, directly mapping all the reflection spectra at various elevation and azimuth angles within the illumination circle $\left(k_{\|}<1.4 k_{0}\right)$ as shown in Fig. $2(\mathrm{~b})$. The size of the obtained image is limited by the $\overline{\mathrm{NA}}$ of our $\mathrm{MO}$ that corresponds to a free-space wave vector of $1.4 k_{0}$. Note that the central dark ring in Fig. 2(b) is the image of the phase ring inside the phase contrast MO and obscures some information, but the information of interest here occurs at larger wave vectors. The bright circle represents the boundary of the illumination at the angle corresponding to total internal reflection (TIR) when the in-plane wave vector of the illumination is $k_{0}$. Outside this TIR circle, SPPs are excited where spatial frequencies satisfy the phasematching condition. Similar to the Kretschmann configuration that uses prisms to excite SPPs on the metal/air interface, in these reflection images reflection dips are observed since the generated SPPs undergo material loss and consequently get dissipated. Therefore, those dark curves outside the TIR circle represent the index ellipsoids of SPPs on the grating in reciprocal space.

As represented by the plasmonic index ellipsoids shown in Fig. 2, the designed deep-subwavelength Au grating can only support propagating SPP waves in a finite azimuthal angular regime centered symmetrically along Au stripes (i.e., the $y$ direction). This plasmonic anisotropy is because the grating acts only as a metal when the state of the polarization is collinear with the axis of the stripes but as a dielectric slab when it is perpendicular to the stripes, consistent with the effective parameters obtained from Eq. (2). Microscopically, the plasmonic anisotropy attributes to the effective dipole moments engineered in an "artificial-atomic" scale with deepsubwavelength features that vary in space depending on the crystal lattice of our grating. Electrons are only free to transport inside the metallic stripes and cannot move very far across the air slit area. Therefore, SPPs can only propagate around the $y \mathrm{di}$ rection. In contrast to SPPs on conventional diffraction metallic gratings [16], however, metal regions between air slits in our metallic nanostructures are too small to support propagating SPP waves along the $x$ direction. The effective dielectric constant changes from positive to negative as the azimuth direction moves from $x$ to $y$, leading to anisotropic SPPs with different in-plane wave vectors in the finite azimuth angular regime around the $y$ axis.

Since SPPs can only be supported in the $y$ direction, a $y$-polarized illumination (TM for SPPs along the metallic stripes) was used to obtain more accurate features and properties of the excited SPP modes on the deep-subwavelength $\mathrm{Au}$ grating with better contrast as shown in Fig. 3. Under the $y$ polarization, the deep-subwavelength $\mathrm{Au}$ grating acts a uniform metal such that only evanescent electromagnetic fields can couple to SPPs. The plasmonic index ellipsoids at the wavelength of $540 \mathrm{~nm}$ shown in Figs. 3(a) and 3(b) can be regarded a part of information in Fig. 2, where reflection intensities with the $x$-polarized optical fields that do not contribute to SPP waves are removed. The same as plasmonic index ellipsoids in Fig. 2, symmetric distribution of SPP modes with respect to the $y$ direction is also mapped as shown by the dark curves outside the TIR circle. The $y$-polarized plasmonic index ellipsoids show the same SPPs as those in Fig. 2 since dark curves are observed at the same in-plane wave vector. Similar plasmonic index ellipsoids are obtained at other wavelengths such as the wavelength of $620 \mathrm{~nm}$ [Figs. 3(c) and 3(d)]. Compared to SPPs at 
(a)

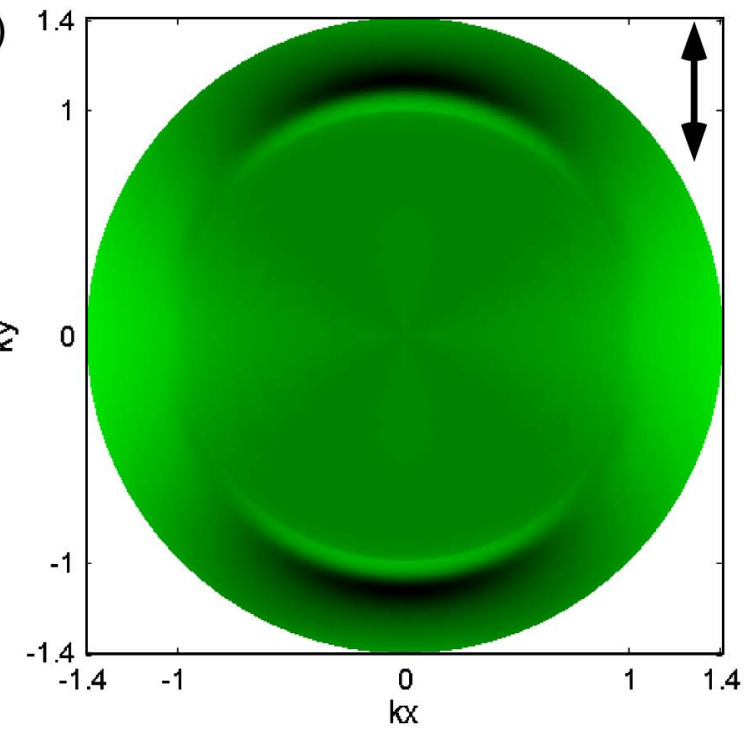

(c)

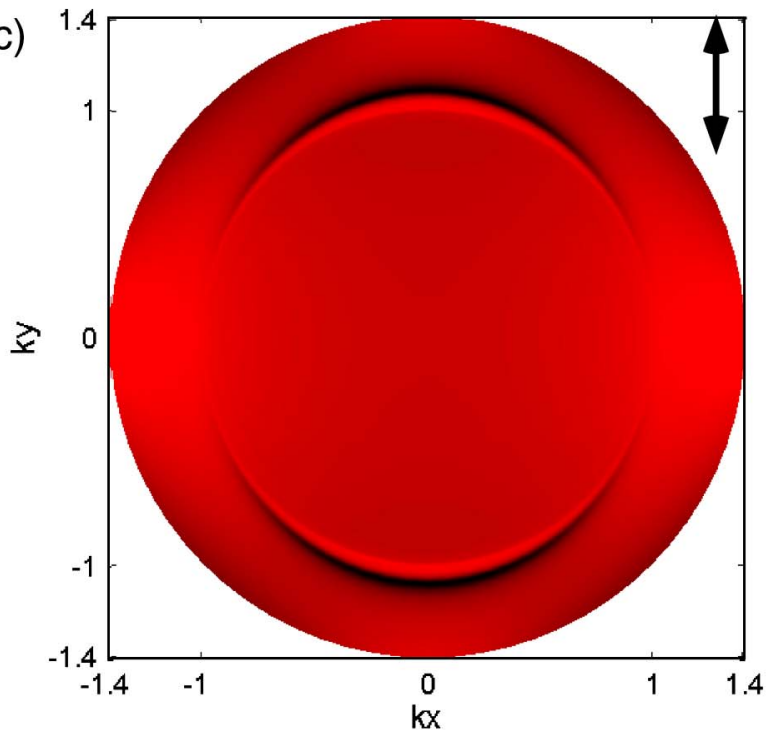

(b)

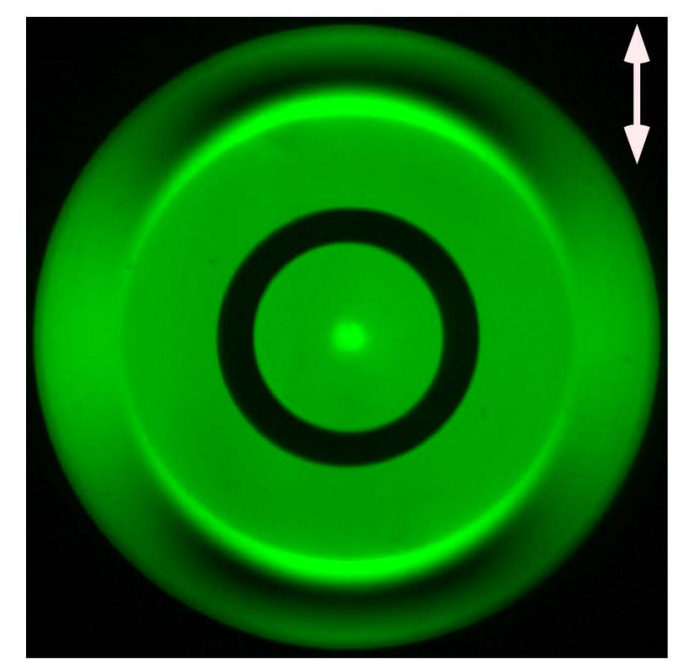

(d)

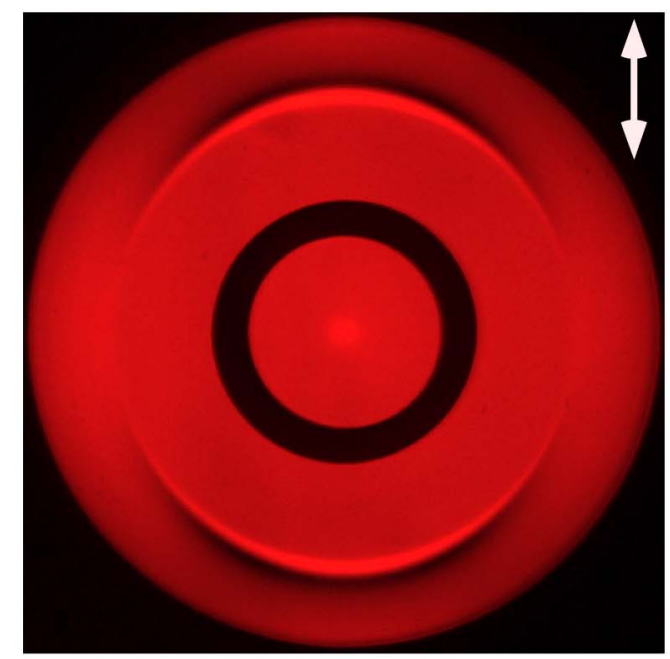

Fig. 3. (Color online) Plasmonic index ellipsoids with the polarization state in the $y$ direction as indicated by arrows. (a) Simulated and (b) measured index ellipsoids of SPPs at the wavelength of $540 \mathrm{~nm}$. (c) Simulated and (d) measured index ellipsoids of SPPs at the wavelength of $620 \mathrm{~nm}$.

the wavelength of $540 \mathrm{~nm}$, due to less material loss from $\mathrm{Au}$, excitation of SPPs at $620 \mathrm{~nm}$ occurs at a smaller in-plane wave vector with a narrower resonant linewidth.

With considering the boundary continuity, the eigenvectors of SPPs on the interface of the deepsubwavelength $\mathrm{Au}$ grating and air along the $y$ axis can be expressed as

$$
k_{\mathrm{spp}}=k_{0} \sqrt{\frac{\varepsilon_{0} \varepsilon_{\perp}}{\varepsilon_{0}+\varepsilon_{\perp}}},
$$

where $k_{0}$ represents the wave vector of light in free space. The dispersion relation of the excited SPPs is shown in Fig. 4(a). The experimentally measured wave vectors from Fig. 3 are integrated for comparison with the analytical calculations using Eq. (3) and numerical simulations in which the wave vectors of SPPs are determined as the positions of reflection dips in the simulated plasmonic index ellipsoids. The index ellipsoids and SPP's dispersion relation of wave vectors are measured as a function of optical wavelengths by analyzing reflection images from 540 to $680 \mathrm{~nm}$. In the range of interest, the experimental and theoretical results are found to be in very good agreement, except for slight discrepancy in theoretical calculations due to neglect of the glass substrate in Eq. (3). Similar to SPPs on a plain metal, SSPs on the grating are associated with larger wave vectors at shorter wavelengths, asymptotically approaching the effective surface plasma frequency. However, the effective plasma frequency of the grating is lower than the original nature plasma frequency of $\mathrm{Au}$ since the electron density is effectively diluted by the air slits.

Figure 4(b) shows a measured broadband index ellipsoid of SPPs on the grating with the polarization 

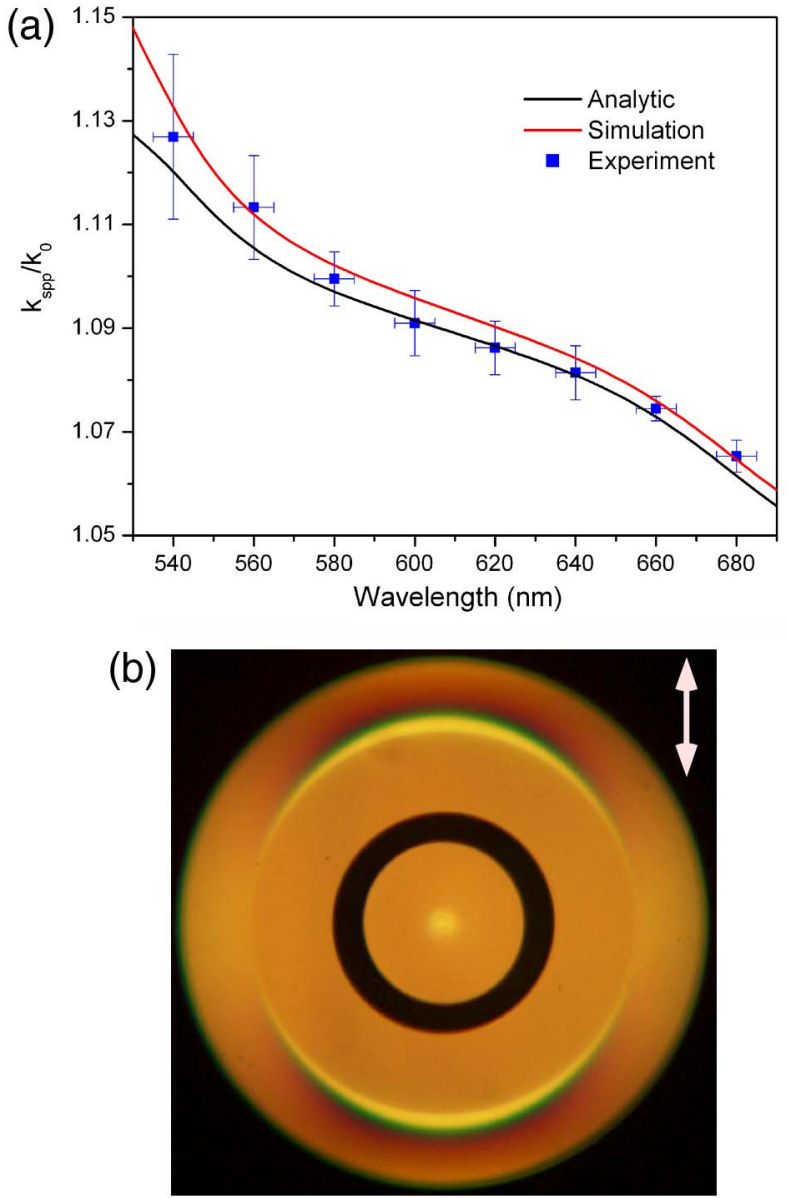

Fig. 4. (Color online) (a) Dispersion relation of SPPs in the $y$ direction on the deep-subwavelength Au grating. Black curve, red curve, and blue dots correspond to the data retrieved from analytical calculations according to Eqs. (1)-(3), numerical simulations, and experimental measurements, respectively. For experimental data, the error bar in wavelengths indicates the bandwidth $(10 \mathrm{~nm})$ of the bandpass filters used in the experimental measurement. (b) Measured plasmonic index ellipsoid with a $y$-polarized broad-band incidence covering all the wavelengths in (a).

state in the $y$ direction as well. Because of its inherent broadband nature, the dispersion relation shown in Fig. 4(a) can be directly observed along the $y$ direction in Fig. 4(b). SPPs excited at different wavelengths are dispersively distributed in reciprocal space, acting as a wavelength disperser and showing a rainbow effect [17]. For example, outside the TIR circle, the inner green curve in reflection corresponds to dissipated SPPs at red wavelengths with relatively smaller in-plane wave vectors, while the outer red curve indicates green SPPs with larger in-plane wave vectors. The dispersion of different colors can be effectively engineered by changing the duty cycle of the grating but keeping the period on a deepsubwavelength nanoscale.

\section{Conclusion}

In conclusion, a deep-subwavelength metallic grating acting as an artificial form-birefringent metal has been constructed and shown to demonstrate different polarizabilities along different optical axes. A plasmonic index ellipsoid is observed and mapped directly in reciprocal space, where SPPs are only excited in a finite azimuth angular regime centered symmetrically along the metallic stripes, demonstrating anisotropic dependence on the polarization state of the fields. A rainbow effect with different colors dispersively distributing in the broadband plasmonic index ellipsoid has also been measured. It is worth noting that the concept of form-birefringent metal is not limited within the specific case shown above. The extension of this work to two and three dimensions also promises novel physics and applications. For example, the reported anisotropic plasmonic metamaterial [14] can be perceived as a combination of two orthogonal sets of deepsubwavelength metallic gratings with different periodicities. Moreover, hybrid plasmonic modes inside the air slits can be excited if the incident polarization and wave vector are not in both $x$ and $y$ directions, enabling strong polarization conversion in plasmonic excitation [15].

This optical plasmonic anisotropy further completes the analogy between plasmonic metamaterials and conventional optical crystals, enabling construction of plasmonic optical devices for applications that rely on chip-scale nanoscale field localization. The effective parameters of these form-birefringent metals are decided by not only the designed geometries but also the index of dielectrics (e.g., air slits in this work). By filling high-index dielectrics in formbirefringent metals, the effective plasma frequency of metals can be manipulated to be lower to the regime of visible/near-IR frequencies. Hence, some exciting SPP phenomena currently demonstrated only in the UV regime such as superlensing [18] can be expected to be realized in a much wider frequency range with the deep-subwavelength engineering of metals. The effective plasma frequency can be further actively controlled if the index of filled dielectrics is tunable. Therefore, form-birefringent metals provide more flexibility in engineering inherent metal properties for complex homogeneous and inhomogeneous plasmonic devices that highly demand a wide-range adiabatic change of metal's dielectric constant and SPP's index in optical circuit designs.

We thank Dr. Dong Yan at UC Riverside for his help in FIB fabrication. This work is supported by the National Science Foundation (NSF), the Defense Advanced Research Projects Agency (DARPA), and NSF Engineering Research Center for Integrated Access Networks.

\section{References}

1. A. Yariv and P. Yeh, Optical Waves in Crystals: Propagation and Control of Laser Radiation (Wiley, 2003).

2. M. Born and E. Wolf, Principles of Optics: Electromagnetic Theory of Propagation, Interference and Diffraction of Light (Cambridge University, 1999).

3. I. Richter, P. C. Sun, F. Xu, and Y. Fainman, "Design consideration of form birefringent microstructures," Appl. Opt. 34 2421-2429 (1995). 
4. R. C. Tyan, A. A. Salvekar, H. P. Chou, C. C. Cheng, A. Scherer, F. Xu, P. C. Sun, and Y. Fainman, "Design, fabrication and characterization of form-birefringent multilayer polarizing beam splitter," J. Opt. Soc. Am. A 14, 1627-1636 (1997).

5. F. Xu, R. C. Tyan, P. C. Sun, Y. Fainman, C. C. Cheng, and A. Scherer, "Form-birefringent computer generated holograms," Opt. Lett. 21, 1513-1515 (1996).

6. U. Levy and Y. Fainman, "Dispersion properties of inhomogeneous nanostructures," J. Opt. Soc. Am. A 21, 881-889 (2004).

7. U. Levy, C. H. Tsai, L. Pang, and Y. Fainman, "Engineering space-variant inhomogeneous media for polarization control," Opt. Lett. 29, 1718-1720 (2004).

8. L. Pang, M. Nezhad, U. Levy, C. H. Tsai, and Y. Fainman, "Form-birefringence structure fabrication in GaAs by use of SU-8 as a dry-etching mask," Appl. Opt. 44, 2377-2381 (2005).

9. A. G. Zhukov and V. I. Smirnov, "Polarizing properties of wire gratings in the long wave infrared," J. Appl. Spectrosc. 3, 304-307 (1965).

10. J. Yao, Z. Liu, Y. Liu, Y. Wang, C. Sun, G. Bartal, A. Stacy, and $\mathrm{X}$. Zhang, "Optical negative refraction in bulk metamaterials," Science 321, 930 (2008).
11. J. Elser, R. Wangberg, V. A. Podolskiy, and E. E. Narimanov, "Nanowire metamaterials with extreme optical anisotropy," Appl. Phys. Lett. 89, 261102 (2006).

12. S. M. Rytov, "Electromagnetic properties of a finely stratified medium," Sov. Phys. JETP 2, 466-475 (1956).

13. E. D. Palik, Handbook of Optical Constants of Solids (Academic, 1985).

14. L. Feng, Z. Liu, V. Lomakin, and Y. Fainman, "Form birefringence metal and its plasmonic anisotropy," Appl. Phys. Lett. 96, 041112 (2010).

15. L. Feng, A. Mizrahi, S. Zamek, Z. Liu, V. Lomakin, and Y. Fainman, "Metamaterials for enhanced polarization conversion in plasmonic excitation," ACS Nano 5, 5100-5106 (2011).

16. R. H. Ritchie, E. T. Arakawa, J. J. Cowan, and R. N. Hamm, "Surface-plasmon resonance effect in grating diffraction," Phys. Rev. Lett. 21, 1530-1533 (1968).

17. Q. Gan, Y. Gao, K. Wagner, D. Vezenov, Y. J. Ding, and F. J. Bartoli, "Experimental verification of the rainbow trapping effect in adiabatic plasmonic gratings," Proc. Natl. Acad. Sci. USA 108, 5169-5173 (2011).

18. N. Fang, H. Lee, C. Sun, and X. Zhang, "Sub-diffractionlimited optical imaging with a silver superlens," Science 308, 534-537 (2005). 